Research Article

\title{
Multiple of Solutions for Nonlocal Elliptic Equations with Critical Exponent Driven by the Fractional $p$-Laplacian of Order $s$
}

\author{
M. Khiddi \\ E.G.A.L, Dépt. Maths, Fac. Sciences, Université Ibn Tofail, BP 133, Kénitre, Morocco \\ Correspondence should be addressed to M. Khiddi; mostapha-2@hotmail.com \\ Received 10 March 2019; Accepted 16 May 2019; Published 28 May 2019 \\ Academic Editor: Chun-Lei Tang
}

Copyright (C) 2019 M. Khiddi. This is an open access article distributed under the Creative Commons Attribution License, which permits unrestricted use, distribution, and reproduction in any medium, provided the original work is properly cited.

In this paper, we study the existence of infinitely many weak solutions for nonlocal elliptic equations with critical exponent driven by the fractional $p$-Laplacian of order $s$. We show the above result when $\lambda>0$ is small enough. We achieve our goal by making use of variational methods, more specifically, the Nehari Manifold and Lusternik-Schnirelmann theory.

\section{Introduction}

This work is concerned with the existence of weak solutions of the following critical fractional $p$-Laplacian problem:

$$
\begin{aligned}
(-\Delta)_{p}^{s} u & =|u|^{p_{s}^{*}-2} u+\lambda|u|^{r-2} u \quad \text { in } \Omega, \\
u & =0 \quad \text { on } \partial \Omega,
\end{aligned}
$$

where $\Omega$ is a smoothly bounded domain of $\mathbb{R}^{N}, N \geq s p, 0<$ $s<1,1<r<p<p_{s}^{*}:=N p /(N-s p)$ is the fractional critical Sobolev exponent, and $\lambda$ is positive parameter.

$(-\Delta)_{p}^{s}$ denotes the fractional $p$-Laplacian operator defined on smooth functions by

$$
\begin{aligned}
& (-\Delta)_{p}^{s} u(x) \\
& =2 \lim _{\varepsilon \searrow 0} \int_{\mathbb{R}^{N} \backslash B_{\varepsilon}(x)} \frac{|u(x)-u(y)|^{p-2}(u(x)-u(y))}{|x-y|^{N+s p}} d y, \\
& x \in \mathbb{R}^{N} .
\end{aligned}
$$

This definition is consistent, up to a normalization constant depending on $N$ and $s$, with the usual definition of the linear fractional Laplacian operator $(-\Delta)^{s}$ when $p=2$. Let us recall the weak formulation of problem (1). Let us set

$$
[u]_{p, s}:=\left(\int_{\mathbb{R}^{2 N}} \frac{|u(x)-u(y)|^{p}}{|x-y|^{N+s p}} d x d y\right)^{1 / p}
$$

by the Gagliardo seminorm of the measurable function $u$ : $\mathbb{R}^{N} \longrightarrow \mathbb{R}$, and let

$$
W^{s, p}\left(\mathbb{R}^{N}\right)=\left\{u \in L^{p}\left(\mathbb{R}^{N}\right):[u]_{p, s}<\infty\right\}
$$

be the fractional Sobolev space endowed with the norm

$$
\|u\|_{s, p}=\left(|u|_{p}^{p}+[u]_{p, s}^{p}\right)^{1 / p},
$$

where $|\cdot|_{p}$ is the norm in $L^{p}\left(\mathbb{R}^{N}\right)$. We work in the closed linear subspace

$$
X_{p}^{s}(\Omega)=\left\{u \in W^{s, p}\left(\mathbb{R}^{N}\right): u=0 \text { a.e. in } \mathbb{R}^{N} \backslash \Omega\right\},
$$

equivalently renormed by setting $\|\cdot\|_{s, p}=[\cdot]_{s, p}$, which is uniformly convex Banach space. We note that the embedding

$$
X_{p}^{s}(\Omega) \hookrightarrow L^{r}(\Omega)
$$

is continuous for $r \in\left[1, p_{s}^{*}\right]$ and compact for $r \in\left[1, p_{s}^{*}\right)$, where $p_{s}^{*}=p N /(N-p s)$ is the fractional critical Sobolev 
exponent (note that when $s=1$ the above exponent reduces to the classical Sobolev exponent $2^{*}$ ). As in the classical case the technical problem posed by such an exponent is that the Sobolev embedding is not compact. Denote by $S_{s, p}$ the best Sobolev constant of the immersion $X_{p}^{s}(\Omega) \hookrightarrow L^{p_{s}^{*}}(\Omega)$, that is,

$$
S_{s, p}:=\inf \left\{\|u\|_{s, p}^{p}:|u|_{p_{s}^{*}}=1, u \in X_{p}^{s}(\Omega)\right\} .
$$

The dual space of $X_{p}^{s}(\Omega)$ is $X_{p}^{s}(\Omega)^{*}$.

Recently, a great attention has been focused on the study of the fractional Laplacian and nonlocal operators of elliptic; this type arises in both pure mathematical research and concrete applications, such as the thin obstacle problem $[1,2]$, minimal surfaces [3], phase transitions [4], crystal dislocation [5], Markov processes [6], and fractional quantum mechanics [7]. This is one of the reasons why nonlocal fractional problems are widely studied in the literature in many different contexts (see [8]).

When $s=1$, our problem becomes a scalar quasilinear elliptic equation as follows:

$$
\begin{aligned}
-\Delta_{p} u & =|u|^{p^{*}-2} u+\lambda|u|^{r-2} u \quad \text { in } \Omega, \\
u & =0 \quad \text { on } \partial \Omega .
\end{aligned}
$$

This has been widely studied by many authors. For example, when $\Omega$ is bounded, the existence of nontrivial solution was studied (see, e.g., $[9,10])$. The typical difficulty in dealing with (9) is that the Sobolev embedding $W_{0}^{1, p}(\Omega) \hookrightarrow L^{p^{*}}(\Omega)$ is not compact, so the usual "Convex-Compact" method does not apply directly. As for the existence of infinitely many solutions to (9), by using Lusternik-Schnirelmans theory, J. G. Azorero and I. P. Aloson proved in [11] that if $\Omega$ is bounded, $1<$ $r<p$, and $\lambda>0$ is small, then (9) has infinitely many solutions. Also the main result of [11] was extended to the equation driven by the operator $-\Delta_{p} u-\Delta_{q} u$ by G. Li and $X$. Liang in [12]. G. M. Figueiredo in [13] generalized the same result of [11] to the elliptic equation generated by the operator $-\operatorname{div}\left(a\left(|\nabla u|^{p}\right)|\nabla u|^{p-2} \nabla u\right)$. Several works have been devoted to study some existence and multiplicity results for fractional problems involving the $p$-Laplacian operator of the type (1), generalizing therefore some classical results obtained in the scalar case. The reader can find a lot of papers in the literature involving this subject; we cite [14-17]. Our goal is to generalize the results of Garcia Azorero and Peral in [11] to the case of the fractional $p$-Laplacian $(-\Delta)_{p}^{s}$ on a bounded domain.

Two major difficulties arise which have to be dealt with in order to reach the desirable conclusions.

First off, it is hard to prove the existence of infinitely many negative energy solutions for our equation by using the variational method because $\mathscr{F}_{\lambda}$ does not satisfy the (PS) conditions, more precisely, because the problem in question incorporates critical exponents.

Secondly, the functional $\mathscr{F}_{\lambda}$ is not bounded from below, so in order to comfortably follow through with our plan, we have to introduce an appropriate truncation to the problem, the choice of which is of utmost importance to the results we get in this paper.
Theorem 1. Assume that $1<r<p<p_{s}^{*}$. Then there exists $\lambda_{0} \in \mathbb{R}_{+}^{*}$ such that, for each $\lambda \in\left(0, \lambda_{0}\right)$, problem (1) has infinitely many solutions with negative energy.

Theorem 1 is new as far as we know and it generalizes a similar result in [11] for the fractional $p$-Laplacian $(-\Delta)_{p}^{s}$ type problem. We mainly follow the way in [11] to prove our main result. The paper is organized as follows. In Section 2, we show that the $(P S)_{c}$ conditions hold for the related energy functional in certain critical levels. That is, we give in a precise range of compactness for the energy functional related. In Section 3, under the assumptions of Theorem 1 and by application of Ljusternik-Schnirelmann methods, we establish the existence of infinitely many solutions with $\lambda>0$ small enough.

\section{The $(P S)_{c}$ Condition for the Associated Functional}

We recall that a weak solution for problem (1) is a function $u: \Omega \longrightarrow \mathbb{R}, u \in X_{p}^{s}(\Omega)$ such that

$$
\begin{aligned}
& \int_{\mathbb{R}^{2 N}} \frac{|u(x)-u(y)|^{p-2}(u(x)-u(y))(\varphi(x)-\varphi(y))}{|x-y|^{N+s p}} d x d y \\
& -\lambda \int_{\Omega}|u|^{r-2} u \varphi d x-\int_{\Omega}|u|^{p_{s}^{*}-2} u \varphi d x=0, \quad \forall \varphi \in X_{p}^{s}(\Omega) .
\end{aligned}
$$

Now let us consider the functional $\mathscr{F}_{\lambda}: X_{p}^{s}(\Omega) \rightarrow \mathbb{R}$ defined by

$$
\mathscr{F}_{\lambda}(u)=\frac{1}{p}\|u\|_{s, p}^{p}-\frac{\lambda}{r}|u|_{r}^{r}-\frac{1}{p_{s}^{*}}|u|_{p_{s}^{*}}^{p_{s}^{*}} .
$$

Note that the functional $\mathscr{F}_{\lambda} \in C^{1}\left(X_{p}^{s}(\Omega), \mathbb{R}\right)$ and its derivative at $u \in X_{p}^{s}(\Omega)$ are given by

$$
\begin{aligned}
& \left\langle\mathscr{F}_{\lambda}^{\prime}(u), \varphi\right\rangle \\
& =\int_{\mathbb{R}^{2 N}} \frac{|u(x)-u(y)|^{p-2}(u(x)-u(y))(\varphi(x)-\varphi(y))}{|x-y|^{N+s p}} d x d y \\
& \quad-\lambda \int_{\Omega}|u|^{r-2} u \varphi d x-\int_{\Omega}|u|^{p_{s}^{*}-2} u \varphi d x,
\end{aligned}
$$

for every $\varphi \in X_{p}^{s}(\Omega)$. Thus, the weak solutions of problem (1) are precisely the critical points of the energy functional $\mathscr{F}_{\lambda}$. Since problem (1) has a variational structure, the proof of the main result (Theorem 1 and its consequences) reduces to finding critical points of the functional by using suitable abstract approaches. As usual in the critical case, the difficulty related to the variational formulation of (1) is the lack of compactness of the injection of the fractional Sobolev space $X_{p}^{s}(\Omega)$ in $L^{p_{s}^{*}}(\Omega)$. To overcome this difficulty in treating problem (1), we show that even if the functional $\mathscr{F}_{\lambda}$ does not verify globally the Palais-Smale condition, it satisfies such a condition in a suitable range related to the best fractional critical Sobolev constant $S_{s, p}$ noted in (8). 
The Nehari manifold associated with $\mathscr{F}_{\lambda}$ is given by

$$
\mathcal{N}_{\lambda}:=\left\{u \in X_{p}^{s}(\Omega) \backslash\{0\}:\left\langle\mathscr{F}_{\lambda}^{\prime}(u), u\right\rangle=0\right\},
$$

where $\mathscr{F}_{\lambda}^{\prime}$ denotes the Gâteaux derivative of $\mathscr{F}_{\lambda}$.

\section{Definition 2.}

(i) For $c \in \mathbb{R}$, a sequence $\left\{u_{n}\right\} \subset X_{p}^{s}(\Omega)$ is a $(P S)_{c}$ for $\mathscr{F}_{\lambda}$ if $\mathscr{F}_{\lambda}\left(u_{n}\right)=c+o(1)$ and $\mathscr{F}_{\lambda}^{\prime}\left(u_{n}\right)=o(1)$ strongly in $X_{p}^{s}(\Omega)^{*}$ as $n \longrightarrow+\infty$, where $X_{p}^{s}(\Omega)^{*}$ is the dual of $X_{p}^{s}(\Omega)$.

(ii) $\mathscr{F}_{\lambda}$ satisfies the $(P S)_{c}$ condition in $X_{p}^{s}(\Omega)$ if any $(P S)_{c}$ sequence for $\mathscr{F}_{\lambda}$ contains a convergent subsequence.

The first step for the $(P S)_{c}$ sequence to hold is bounded.

Lemma 3. Let $c \in \mathbb{R}$. If $\left\{u_{n}\right\}$ is $(P S)_{c}$ - sequence for $\mathscr{F}_{\lambda}$, then $\left\{u_{n}\right\}$ is bounded in $X_{p}^{s}(\Omega)$.

Proposition 4. There exists a $K>0$ such that, for any $\lambda>0$ and

$$
c \leq \frac{s}{N} S_{s, p}^{N / s p}-K \lambda^{p_{s}^{*} / p_{s}^{*}-r},
$$

the functional $\mathscr{F}_{\lambda}$ satisfies $(P S)_{c}$ condition.

Proof. Let $\left\{u_{n}\right\}$ be a sequence in $X_{p}^{s}(\Omega)$ such that

$$
\begin{aligned}
& \mathscr{F}_{\lambda}\left(u_{n}\right)=\frac{1}{p}\left\|u_{n}\right\|_{s, p}^{p}-\frac{\lambda}{r}\left|u_{n}\right|_{r}^{r}-\frac{1}{p_{s}^{*}}\left|u_{n}\right|_{p_{s}^{*}}^{p_{s}^{*}}=c+o(1), \\
& \left\langle\mathscr{F}_{\lambda}^{\prime}\left(u_{n}\right), v\right\rangle \\
& =\int_{\mathbb{R}^{2 N}} \frac{\left|u_{n}(x)-u_{n}(y)\right|^{p-2}\left(u_{n}(x)-u_{n}(y)\right)(v(x)-v(y))}{|x-y|^{N+s p}} d x d y \\
& \quad-\lambda \int_{\Omega}\left|u_{n}\right|^{r-2} u_{n} v d x-\int_{\Omega}\left|u_{n}\right|^{p_{s}^{*}-2} u_{n} v d x=o\left(\left\|u_{n}\right\|_{s, p}\right),
\end{aligned}
$$

as $n \longrightarrow \infty$, for all $v \in X_{p}^{s}(\Omega)$. Then

$$
\begin{aligned}
& \frac{s}{N}\left|u_{n}\right|_{p_{s}^{*}}^{p_{s}^{*}}-\frac{1}{\lambda}\left(\frac{1}{r}-\frac{1}{p}\right)\left|u_{n}\right|_{r}^{r} \\
& =\mathscr{F}_{\lambda}\left(u_{n}\right)-\frac{1}{p}\left\langle\mathscr{F}_{\lambda}^{\prime}\left(u_{n}\right), u_{n}\right\rangle \\
& =o\left(\left\|u_{n}\right\|_{s, p}\right)+O(1) .
\end{aligned}
$$

From (17) and the Hölder inequality, it is implied that $\left(u_{n}\right)$ is bounded in $X_{p}^{s}(\Omega)$. Up to a subsequence, this implies the following:

$$
\begin{aligned}
& u_{n} \rightarrow u \text { in } X_{p}^{s}(\Omega), \\
& u_{n}(x) \longrightarrow u(x) \text { a.e in } \Omega, \\
& \left|u_{n}(x)-u_{n}(y)\right|^{p-2}\left(u_{n}(x)-u_{n}(y)\right) / \mid x- \\
& \left.y\right|^{N+s p / p^{\prime}} \text { is bounded in } L^{p^{\prime}}\left(\mathbb{R}^{2 N}\right), \\
& u_{n} \longrightarrow u \text { in } L^{r}(\Omega), \quad 1 \leq r<p_{s}^{*},
\end{aligned}
$$

$$
\begin{aligned}
& \left|u_{n}(x)-u_{n}(y)\right|^{p-2}\left(u_{n}(x)-u_{n}(y)\right) / \mid x- \\
& \left.y\right|^{N+s p / p^{\prime}} \text { is bounded in } L^{p^{\prime}}\left(\mathbb{R}^{2 N}\right) \text { and converges to } \\
& |u(x)-u(y)|^{p-2}(u(x)-u(y)) / \mid x- \\
& \left.y\right|^{N+s p / p^{\prime}} \text {, a.e in } \mathbb{R}^{2 N} \text {. }
\end{aligned}
$$

So

$$
\begin{aligned}
& \int_{\mathbb{R}^{2 N}} \frac{\left|u_{n}(x)-u_{n}(y)\right|^{p-2}\left(u_{n}(x)-u_{n}(y)\right)(v(x)-v(y))}{|x-y|^{N+s p}} d x d y \\
& \longrightarrow \int_{\mathbb{R}^{2 N}} \frac{|u(x)-u(y)|^{p-2}(u(x)-u(y))(v(x)-v(y))}{|x-y|^{N+s p}} d x d y
\end{aligned}
$$

as $n \longrightarrow \infty$.

Moreover

$$
\begin{aligned}
\int_{\Omega}\left|u_{n}\right|^{r-2} u_{n} v d x & \longrightarrow \int_{\Omega}|u|^{r-2} u v d x \\
\text { and } \int_{\Omega}\left|u_{n}\right|^{p_{s}^{*}-2} u_{n} v d x & \longrightarrow \int_{\Omega}|u|^{p_{s}^{*}-2} u v d x
\end{aligned}
$$

So passing to the limit in (16) shows that $u \in X_{p}^{s}(\Omega)$ is a weak solution of (1). Setting $v_{n}=u_{n}-u$, we have

$$
\left\|v_{n}\right\|_{s, p}^{p}=\left\|u_{n}\right\|_{s, p}^{p}-\|u\|_{s, p}^{p}+o(1)
$$

By Brezis-Lieb’s Lemma [18], we get

$$
\begin{aligned}
\left|v_{n}\right|_{p_{s}^{*}}^{p_{s}^{*}} & =\left|u_{n}\right|_{p_{s}^{*}}^{p_{s}^{*}}-|u|_{p_{s}^{*}}^{p_{s}^{*}}+o(1) . \\
c+o(1)= & \frac{1}{p}\left\|v_{n}\right\|_{s, p}^{p}+\frac{1}{p}\|u\|_{s, p}^{p}-\frac{1}{p_{s}^{*}}\left|v_{n}\right|_{p_{s}^{*}}^{p_{s}^{*}} \\
& -\frac{1}{p_{s}^{*}}|u|_{p_{s}^{*}}^{p_{s}^{*}}-\frac{\lambda}{r}|u|_{r}^{r},
\end{aligned}
$$

as $n \longrightarrow+\infty$. Taking $v=u_{n}$ in (16) and Brezis-Liebs Lemma again, we have

$$
\left\|v_{n}\right\|_{s, p}^{p}=\lambda|u|_{r}^{r}+\left|v_{n}\right|_{p_{s}^{*}}^{p_{s}^{*}}+|u|_{p_{s}^{*}}^{p_{s}^{*}}+o(1)
$$

Since $u_{n}$ is bounded in $X_{p}^{s}(\Omega)$ and converges to $u$ in $L^{p}(\Omega)$, testing (16) with $v=u$ gives

$$
\|u\|_{s, p}^{p}=\lambda|u|_{r}^{r}+|u|_{p_{s}^{*}}^{p_{s}^{*}} .
$$

It follows from (23) and (24) that

$$
\left\|v_{n}\right\|_{s, p}^{p}=\left.\left|v_{n}\right|\right|_{p_{s}^{*}} ^{P_{s}^{*}}+o(1)
$$

We suppose that

$$
\lim _{n \longrightarrow \infty}\left\|v_{n}\right\|_{s, p}^{p}=l=\lim _{n \longrightarrow \infty}\left|v_{n}\right|_{p_{s}^{*}}^{p_{s}^{*}}
$$

By the definition of the best constant $S_{s, p}$ given in (8), we have

$$
\left\|v_{n}\right\|_{s, p}^{p} \geq S_{s, p}\left|v_{n}\right|_{p_{s}^{*}}^{p},
$$


so

$$
l \geq S_{s, p} l^{p / p_{s}^{*}}
$$

If $l=0$, then the lemma is proved. If $l>0$, then (28) implies that

$$
l \geq S_{s, p}^{N / s p}
$$

From (29) and (22), we have

$$
\begin{aligned}
c= & \frac{1}{p} l+\frac{1}{p}\|u\|_{s, p}^{p}-\frac{1}{p_{s}^{*}} l-\frac{1}{p_{s}^{*}}|u|_{p_{s}^{*}}^{p_{s}^{*}}-\frac{\lambda}{r}|u|_{r}^{r} \\
= & \frac{s}{N} l+\left(\frac{1}{p}-\frac{1}{p_{s}^{*}}\right)|u|_{p_{s}^{*}}^{p_{s}^{*}}+\lambda\left(\frac{1}{p}-\frac{1}{r}\right)|u|_{r}^{r} \\
\geq & \frac{s}{N} S_{s, p}^{N / s p}+\frac{s}{N}|u|_{p_{s}^{*}}^{p_{s}^{*}}+\lambda\left(\frac{1}{p}-\frac{1}{r}\right)|u|_{r}^{r} \\
\geq & \frac{s}{N} S_{s, p}^{N / s p}+\frac{s}{N}|u|_{p_{s}^{*}}^{p_{s}^{*}} \\
& +\lambda\left(\frac{1}{p}-\frac{1}{r}\right)|\Omega|^{p_{s}^{*}-r / p_{s}^{*}}|u|_{p_{s}^{*}}^{r} .
\end{aligned}
$$

We consider the following function $f(x)=$ $(s / N) x^{p_{s}^{*}}-\lambda(1 / r-1 / p)|\Omega|^{p_{s}^{*}-r / p_{s}^{*}} x^{r}$. This function obtains this absolute minimum for $x>0$ at point $x_{0}=\left(\lambda\left((p-r) /\left(p_{s}^{*}-p\right)\right)|\Omega|^{p_{s}^{*}-r / p_{s}^{*}}\right)^{1 / p_{s}^{*}-r}$, that is,

$$
\begin{aligned}
& f(x) \geq f\left(x_{0}\right)=-\lambda^{p_{s}^{*} / p_{s}^{*}-r}|\Omega|\left(\frac{p-r}{p_{s}^{*}-p}\right)^{r /\left(p_{s}^{*}-r\right)} \\
& \cdot\left(\frac{p-r}{p}\right)\left(\frac{p_{s}^{*}-r}{r p_{s}^{*}}\right)
\end{aligned}
$$

and let the constant $K=|\Omega|\left((p-r) /\left(p_{s}^{*}-p\right)\right)^{r /\left(p_{s}^{*}-r\right)}((p-$ $r) / p)\left(\left(p_{s}^{*}-r\right) / r p_{s}^{*}\right)$ be strictly positive because $1<r<p<$ $p_{s}^{*}$.

$$
\begin{aligned}
c & \geq \frac{s}{N} S_{s, p}^{N / s p}+\frac{s}{N}|u|_{p_{s}^{*}}^{p_{s}^{*}}+\lambda\left(\frac{1}{p}-\frac{1}{r}\right)|\Omega|^{p_{s}^{*}-r / p_{s}^{*}}|u|_{p_{s}^{*}}^{r}, \\
& \geq \frac{s}{N} S_{s, p}^{N / s p}-K \lambda^{p_{s}^{*} / p_{s}^{*}-r} .
\end{aligned}
$$

This leads to a contradiction with (9). Therefore $l=0$ and the proof is complete.

\section{Proof of the Main Result}

Under the hypothesis $1<r<p<N$, using Sobolev's inequality we obtain

$$
\mathscr{F}_{\lambda}(u) \geq h\left(\|u\|_{s, p}\right)
$$

where

$$
h(x)=\frac{1}{p} x^{p}-\frac{1}{p_{s}^{*} S_{s, p}^{p_{s}^{*} / p}} x^{p_{s}^{*}}-\frac{\lambda}{r} C_{p, r} x^{r}
$$

and where $C_{r, p}$ is a positive constant independent of $u \in$ $X_{p}^{s}(\Omega)$. An easy computation shows that, for all $0<\lambda<\lambda_{0}=$ $C_{p, r}^{-1}\left(\left(p_{s}^{*}-p\right) /\left(p_{s}^{*}-r\right)\right)\left[\left((p-r) /\left(p_{s}^{*}-r\right)\right) S_{s, p}^{p_{s}^{*} / p}\right]^{(p-r) /\left(p_{s}^{*}-p\right)}$, the real valued function $x \longmapsto h(x)$ has exactly two positive zeros denoted by $R_{0}$ and $R_{1}$, and the point $R$ is where $h$ attains its nonnegative maximum and verifies $R_{0}<R<R_{1}$.

We now introduce the following truncation of the functional $\mathscr{F}_{\lambda}$. Take the nonincreasing function $\tau: \mathbb{R}^{+} \longrightarrow[0,1]$ and $C^{\infty}\left(\mathbb{R}^{+}\right)$such that

$$
\begin{array}{ll}
\tau(x)=1 & \text { if } x \leq R_{0}, \\
\tau(x)=0 & \text { if } x \geq R_{1} .
\end{array}
$$

Let $\varphi(u)=\tau\left(\|u\|_{s, p}\right)$. We consider the truncated functional

$$
\begin{aligned}
\widetilde{\mathscr{F}}_{\lambda}(u)= & \frac{1}{p}\|u\|_{s, p}^{p}-\frac{\lambda}{r} \int_{\Omega}|u|^{r} d x \\
& -\frac{1}{p_{s}^{*}} \int_{\Omega}|u|^{p_{s}^{*}} \varphi(u) d x .
\end{aligned}
$$

Similar to (33), we have

$$
\widetilde{\mathscr{F}}_{\lambda}(u) \geq \bar{h}\left(\|u\|_{s, p}\right)
$$

where

$$
\bar{h}(x)=\frac{1}{p} x^{p}-\frac{1}{p_{s}^{*} S_{s, p}^{p_{s}^{*} / p}} x^{p_{s}^{*}} \tau(x)-\frac{\lambda}{r} C_{p, r} x^{r}
$$

Clearly,

$$
\bar{h}(x) \geq h(x)
$$

for $x \geq 0$ and $\bar{h}(x)=h(x)$ if $0 \leq x \leq R_{0}, \bar{h}(x) \geq 0$, if $R_{0}<x \leq$ $R_{1}$ and if $x>R_{1}, \bar{h}(x)=x^{r}\left((1 / p) x^{p-r}-(\lambda / r) C_{p, r}\right)$ is strictly increasing and so $\bar{h}(x)>0$, if $x>R_{1}$. Consequently

$$
\bar{h}(x) \geq 0 \text { for } x \geq R_{0} .
$$

We have the following result.

Lemma 5. This lemma can be expressed as three assertions:

(1) $\widetilde{\mathscr{F}}_{\lambda} \in \mathscr{C}^{1}\left(X_{p}^{s}(\Omega), \mathbb{R}\right)$ is even.

(2) If $\widetilde{\mathscr{F}}_{\lambda}\left(u_{0}\right) \leq 0$ then $\left\|u_{0}\right\|_{s, p}<R_{0}$. Moreover, $\widetilde{\mathscr{F}}_{\lambda}(u)=\mathscr{F}_{\lambda}(u)$ for all $u$ in a small enough neighborhood of $u_{0}$.

(3) There exists $\lambda_{0}>0$, such that if $0<\lambda<\lambda_{0}$, then $\widetilde{\mathscr{F}}_{\lambda}$ verifies a local Palais-Smale condition for $c \leq 0$.

Proof. Since $\varphi \in \mathscr{C}^{\infty}$ and $\varphi(u)=1$ for $u$ near $0, \widetilde{\mathscr{F}}_{\lambda} \epsilon$ $\mathscr{C}^{1}\left(X_{p}^{s}(\Omega), \mathbb{R}\right)$ and assertion (1) holds.

By taking $\widetilde{\mathscr{F}}_{\lambda}\left(u_{0}\right) \leq 0$, we can deduce from (37) that

$$
\bar{h}\left(\left\|u_{0}\right\|_{s, p}\right) \leq 0
$$


and by (40) and (41) we have

$$
\left\|u_{0}\right\|_{s, p}<R_{0}
$$

and (2) holds.

For the proof of (3), let $\left\{u_{n}\right\} \subset X_{p}^{s}(\Omega)$ be a $(P S)_{c}$ sequence $\widetilde{\mathscr{F}}_{\lambda}$, with $c<0$. Then we may assume that $\widetilde{\mathscr{F}}_{\lambda}\left(u_{n}\right)<0$, $\widetilde{F}_{\lambda}^{\prime}\left(u_{n}\right) \longrightarrow 0$. By (8) in Lemma 5 there exists $\lambda_{0}>0$ such that $0<\lambda<\lambda_{0},\left\|u_{n}\right\|_{s, p}<R_{0}$, so $\widetilde{\mathscr{F}}_{\lambda}\left(u_{n}\right)=\mathscr{F}_{\lambda}\left(u_{n}\right)$ and $\widetilde{\mathscr{F}}_{\lambda}^{\prime}\left(u_{n}\right)=\mathscr{F}_{\lambda}^{\prime}\left(u_{n}\right)$. By Proposition $4, \mathscr{F}_{\lambda}$ satisfies $(P S)_{c}$ condition for $c<0$, so there is a subsequence $\left\{u_{n}\right\}$ such that $u_{n} \longrightarrow u$ in $X_{p}^{s}(\Omega)$. Thus $\widetilde{\mathscr{F}}_{\lambda}$ satisfies $(P S)_{c}$ condition for $c<0$.

We will use the genus of symmetric set in $X_{p}^{s}(\Omega)$, where the genus $\gamma(A)$ is the smallest integer $m$, such that there exists an odd map

$$
\phi \in \mathscr{C}\left(A, \mathbb{R}^{m} \backslash\{0\}\right),
$$

where $A$ is a closed symmetric set in $X$ that does not contain zero (see [19]).

It is possible to prove the existence of level sets of $\widetilde{I}_{\lambda}$ with arbitrarily large genus, more precisely,

Lemma 6. $\forall n \in \mathbb{N} \exists e(n)>0$ such that

$$
\gamma\left(\left\{u \in X_{p}^{s}(\Omega): \widetilde{\mathscr{F}}_{\lambda}(u) \leq-\epsilon(n)\right\}\right) \geq n .
$$

Proof. Let $n \in \mathbb{N}$. we consider $E_{n}$ to be subspaces of $X_{p}^{s}(\Omega)$ with $E_{n}$ being an n-dimensional subspace of $X_{p}^{s}(\Omega)$. Let $\left\{u_{n}\right\} \in E_{n}$ with norm $\left\|u_{n}\right\|_{s, p}=1$. For $0<\rho<\overline{R_{0}}$

$$
\begin{aligned}
\widetilde{\mathscr{F}}_{\lambda}\left(\rho u_{n}\right) \leq & \frac{1}{p} \rho^{p}-\frac{\rho^{p_{s}^{*}}}{p_{s}^{*}} \int_{\Omega}\left|u_{n}\right|^{p_{s}^{*}} d x \\
& -\frac{\lambda}{r} \rho^{r} \int_{\Omega}\left|u_{n}\right|^{r} d x
\end{aligned}
$$

we define

$$
\alpha_{n}:=\inf \left\{\int_{\Omega}\left|u_{n}\right|^{p_{s}^{*}} d x: u_{n} \in E_{n},\left\|u_{n}\right\|_{s, p}=1\right\}>0,
$$

and

$$
\beta_{n}:=\inf \left\{\int_{\Omega}\left|u_{n}\right|^{r}: u_{n} \in E_{n},\left\|u_{n}\right\|_{s, p}=1\right\}>0 .
$$

By using the definitions of $\alpha_{n}, \beta_{n}$, and inequality (45), we obtain

$$
\widetilde{\mathscr{F}}_{\lambda}\left(\rho u_{n}\right) \leq \frac{1}{p} \rho^{p}-\frac{\alpha_{n}}{p_{s}^{*}} \rho^{p_{s}^{*}}-\frac{\lambda \beta_{n}}{r} \rho^{r} .
$$

Then, there exists $\epsilon(n)>0$ and $0<\rho<R_{0}$ such that

$$
\widetilde{\mathscr{F}}_{\lambda}(\rho u) \leq-\epsilon(n)
$$

for $u \in E_{n}$ and $\left\|u_{n}\right\|=1$.

$$
\begin{aligned}
\text { Let } S_{\eta}=\left\{u \in X_{p}^{s}(\Omega) /\|u\|=\eta\right\} \text {, so } \\
\qquad S_{\eta} \cap E_{n} \subset\left\{u \in X_{p}^{s}(\Omega) / \widetilde{F}_{\lambda}(u) \leq-\epsilon(n)\right\},
\end{aligned}
$$

therefore, by the properties of the genus (see [19])

$$
\gamma\left(\left\{u \in X_{p}^{s}(\Omega) / \widetilde{\mathscr{F}}_{\lambda}(u) \leq-\epsilon\right\}\right) \geq \gamma\left(S_{\eta} \cap E_{n}\right) \geq n .
$$

We are now in a position to prove our main result.

Proof of Theorem 1. For $n \in \mathbb{N}$, we define

$$
\begin{aligned}
\Gamma_{n} & =\left\{A \subset X_{p}^{s}(\Omega)-\{0\} / A \text { is close, } A=-A, \gamma(A)\right. \\
& \geq n\} .
\end{aligned}
$$

Let us set

$$
c_{n}=\min _{A \in \Gamma_{n}} \max _{u \in A} \widetilde{I}_{\lambda}(u),
$$

and

$$
K_{c}=\left\{u \in X_{p}^{s}(\Omega): \widetilde{\mathscr{F}}_{\lambda}^{\prime}(u)=0, \widetilde{\mathscr{F}}_{\lambda}(u)=c\right\},
$$

and suppose $0<\lambda<\lambda_{o}$, where $\lambda_{0}$ is the constant given by Lemma 5.

$$
\widetilde{\mathscr{F}}_{\lambda}^{-\epsilon}=\left\{u \in X_{p}^{s}(\Omega) / \widetilde{\mathscr{F}}_{\lambda}(u) \leq-\epsilon\right\} .
$$

By Lemma 6 there exists $\epsilon(n)>0$ such that $\gamma\left(\widetilde{\mathscr{F}}_{\lambda}^{-\epsilon}\right) \geq n$, for all $n \in \mathbb{N}$. Because $\widetilde{\mathscr{F}}_{\lambda}(u)$ is continuous and even, $\widetilde{\mathscr{F}}_{\lambda}^{-\epsilon} \in \Gamma_{n}$, then $c_{n} \leq-\epsilon(n)<0$ for all $\mathrm{n}$ in $\mathbb{N}$. But $\widetilde{\mathscr{F}}_{\lambda}$ is bounded from below; hence $c_{n}>-\infty$ for all $\mathrm{n}$ in $\mathbb{N}$.

Let us assume that $c=c_{n}=c_{n+1}=\cdots=c_{n+r}$. Note that $c<0$; therefore, $\widetilde{\mathscr{F}}_{\lambda}$ verifies the Plais-Smale condition in $c$, and it is easy to see that $K_{c}$ is a compact set.

If $\gamma\left(K_{c}\right) \leq r$, there exists a closed and symmetric set $\mathrm{U}$ verifying $K_{c} \subset U$, such that $\gamma(U) \leq r$. By the deformation lemma (see [20]), we have an odd homeomorphism $\eta: X \longrightarrow$ $X$, such that $\eta\left(\widetilde{\mathscr{F}}_{\lambda}^{c+\delta}-U\right) \subset \widetilde{\mathscr{F}}_{\lambda}^{c-\delta}$, for some $\delta>0$. By definition,

$$
c=c_{n}=\inf _{A \in \Gamma_{n+r}} \sup _{u \in A} \widetilde{\mathscr{F}}_{\lambda}(u) .
$$

There exists then $A \in \Gamma_{n+r}$, such that $\sup _{u \in A} \widetilde{\mathscr{F}}_{\lambda}(u)<c+\delta$, i.e., $A \subset \widetilde{\mathscr{F}}_{\lambda}^{c+\delta}$,

$$
\eta(A-U) \subset \eta\left(\widetilde{\mathscr{F}}_{\lambda}^{c+\delta}-U\right) \subset \widetilde{\mathscr{F}}_{\lambda}^{c-\delta} .
$$

But $\gamma(\overline{A-U}) \geq \gamma(A)-\gamma(U) \geq n$, and $\gamma(\eta(\overline{A-U})) \geq$ $\gamma(\overline{A-U})) \geq n$.

Then, $\eta(\overline{A-U}) \in \Gamma_{n}$. And this is a contradiction; in fact,

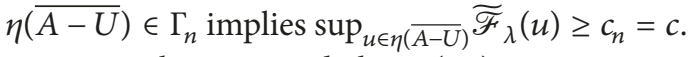

So we have proved that $\gamma\left(K_{c}\right) \geq r+1$. We are now ready to show that $\mathscr{F}_{\lambda}$ has infinitely many critical point 
solutions. Note that $c_{n}$ is nondecreasing and strictly negative. We distinguish two cases.

Case 1. Suppose that there are $1<n_{1}<\cdots n_{i}<\cdots$, satisfying

$$
c_{n_{1}}<\cdots<c_{n_{i}}<\cdots .
$$

In this case, we have infinitely many distinct critical points.

Case 2. We assume in this case that, for some positive integer $n_{0}$, there is $r \geq 1$ such that $c=c_{n_{0}}=c_{n_{0}+1}=\cdots=c_{n_{0}+r}$; then $\gamma\left(K_{c_{n_{0}}}\right) \geq r+1$ which shows that $K_{c_{n_{0}}}$ contains infinitely many distinct elements. Since $\widetilde{\mathscr{F}}_{\lambda}(u)=\mathscr{F}_{\lambda}(u)$ if $\widetilde{\mathscr{F}}_{\lambda}(u)<0$, we see that there are infinitely many critical points of $\mathscr{F}_{\lambda}(u)$. The theorem is proved.

\section{Data Availability}

No data were used to support this study.

\section{Conflicts of Interest}

The author declares no conflicts of interest.

\section{References}

[1] L. A. Caffarelli, S. Salsa, and L. Silvestre, "Regularity estimates for the solution and the free boundary of the obstacle problem for the fractional Laplacian," Inventiones Mathematicae, vol. 171, no. 2, pp. 425-461, 2008.

[2] L. Silvestre, "Regularity of the obstacle problem for a fractional power of the Laplace operator," Communications on Pure and Applied Mathematics, vol. 60, no. 1, pp. 67-112, 2007.

[3] L. Caffarelli, J.-M. Roquejoffre, and O. Savin, "Nonlocal minimal surfaces," Communications on Pure and Applied Mathematics, vol. 63, no. 9, pp. 1111-1144, 2010.

[4] Y. Sire and E. Valdinoci, "Fractional Laplacian phase transitions and boundary reactions: A geometric inequality and a symmetry result," Journal of Functional Analysis, vol. 256, no. 6, pp. 1842-1864, 2009.

[5] Y. H. Wei and X. F. Su, "Multiplicity of solutions for non-local elliptic equations driven by the fractional Laplacian," Calculus of Variations and Partial Differential Equations, vol. 52, no. 1-2, pp. 95-124, 2015

[6] Q.-Y. Guan and Z.-M. Ma, "Boundary problems for fractional Laplacians," Stochastics and Dynamics, vol. 5, no. 3, pp. 385-424, 2005.

[7] N. Laskin, "Fractional quantum mechanics and Lévy path integrals," Physics Letters A, vol. 268, no. 4-6, pp. 298-305, 2000.

[8] E. Di Nezza, G. Palatucci, and E. Valdinoci, "Hitchhiker's guide to the fractional Sobolev spaces," Bulletin des Sciences Mathématiques, vol. 136, no. 5, pp. 521-573, 2012.

[9] Z. Xiping, "Nontrivial solution of quasilinear elliptic equations involving critical Sobolev exponent," Science in China Series AMathematics, Physics, Astronomy \& Technological Science, vol. 31, no. 10, pp. 1166-1181, 1988.

[10] G. B. Li, The Existence of Nontrivial Solution of Quasilinear Elliptic Pde of Variational Type, Wuhan University, Hubei, China, 1987.
[11] J. Garcia Azorero and I. Peral Alonso, "Multiplicity of solutions for elliptic problems with critical exponent or with a nonsymmetric term," Transactions of the American Mathematical Society, vol. 323, no. 2, pp. 877-895, 1991.

[12] L. Gongbao and Z. Guo, "Multiple solutions for the p\&qLaplacian problem with critical exponent," Acta Mathematica Scientia, vol. 29, no. 4, pp. 903-918, 2009.

[13] G. M. Figueiredo, "Existence and multiplicity of solutions for a class of $\mathrm{p} \& \mathrm{q}$ elliptic problems with critical exponent," Mathematische Nachrichten, vol. 286, no. 11-12, pp. 1129-1141, 2013.

[14] L. Brasco and M. Squassina, "Optimal solvability for a nonlocal problem at critical growth," Journal of Differential Equations, vol. 264, no. 3, pp. 2242-2269, 2018.

[15] K. Perera, M. Squassina, and Y. Yang, "Bifurcation and multiplicity results for critical fractional p-Laplacian problems," Mathematische Nachrichten, vol. 289, no. 2-3, pp. 332-342, 2016.

[16] J. Mawhin and G. Molica Bisci, "A Brezis-Nirenberg type result for a nonlocal fractional operator," Journal of The London Mathematical Society-Second Series, vol. 95, no. 1, pp. 73-93, 2017.

[17] R. Servadei and E. Valdinoci, "The Brezis-Nirenberg result for the fractional Laplacian," Transactions of the American Mathematical Society, vol. 367, no. 1, pp. 67-102, 2015.

[18] H. Brézis and E. Lieb, "A relation between pointwise convergence of functions and convergence of functionals," Proceedings of the American Mathematical Society, vol. 88, no. 3, pp. 486490, 1983.

[19] O. Kavian, Introduction à la théorie des points critiques et applications aux problèmes elliptiques, Springer-Verlag, Paris, France, 1993.

[20] M. Struwe, Variational Methods: Applications to Nonlinear Partial Differential Equations and Hamiltonian Systems, Springer, Berlin, Germany, 3rd edition, 1990. 


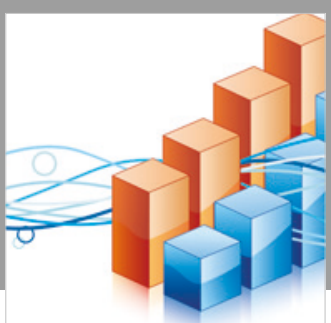

Advances in

Operations Research

\section{-n-m}
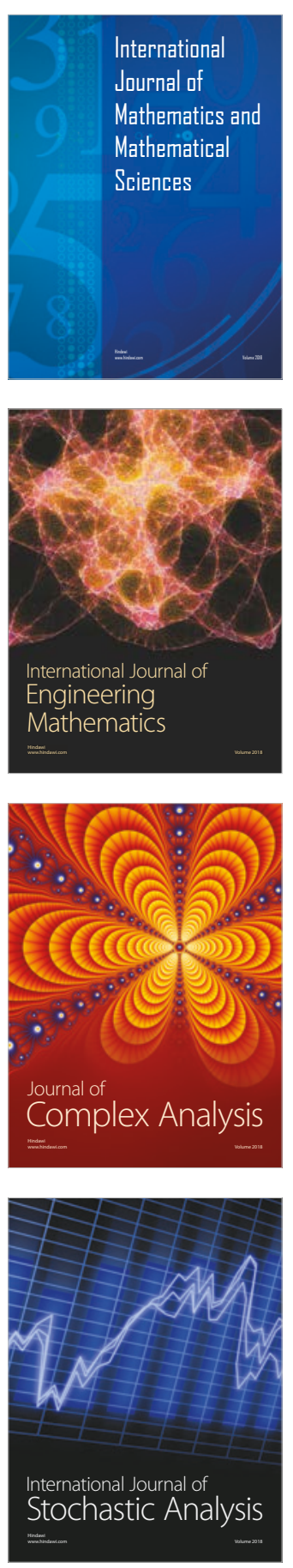
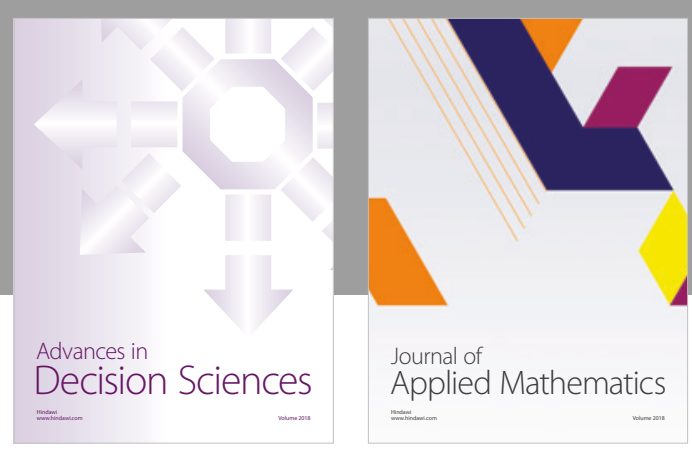

Journal of

Applied Mathematics
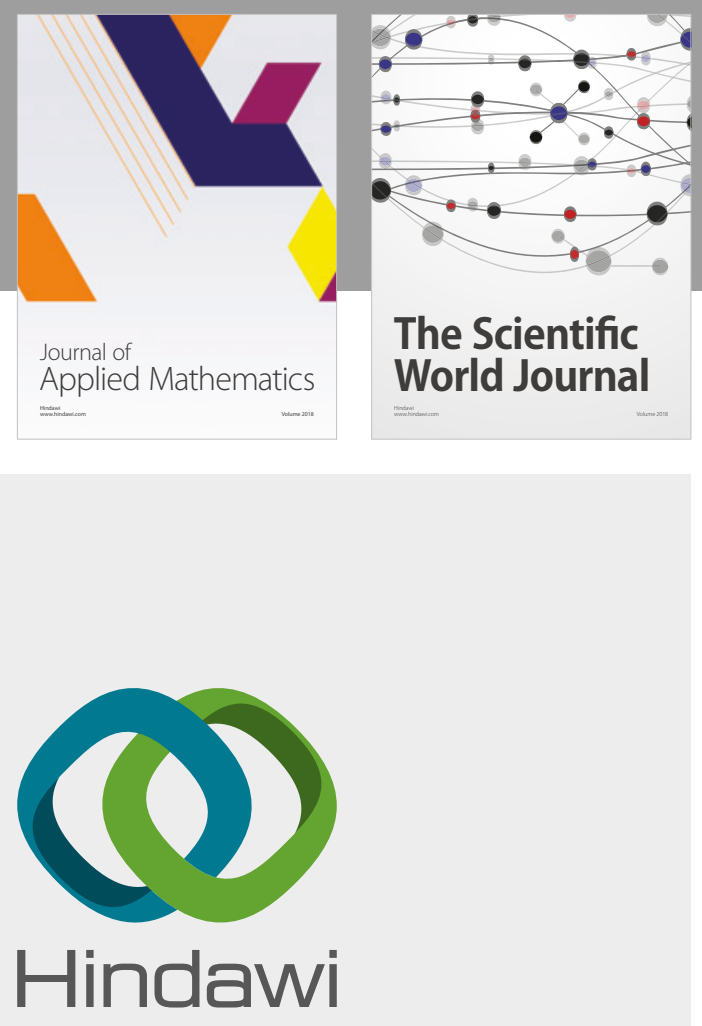

Submit your manuscripts at

www.hindawi.com

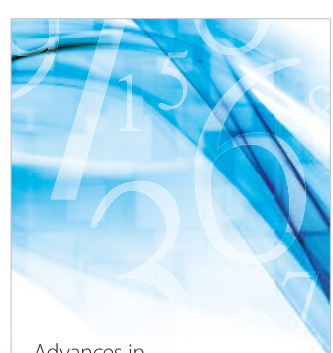

Advances in
Numerical Analysis
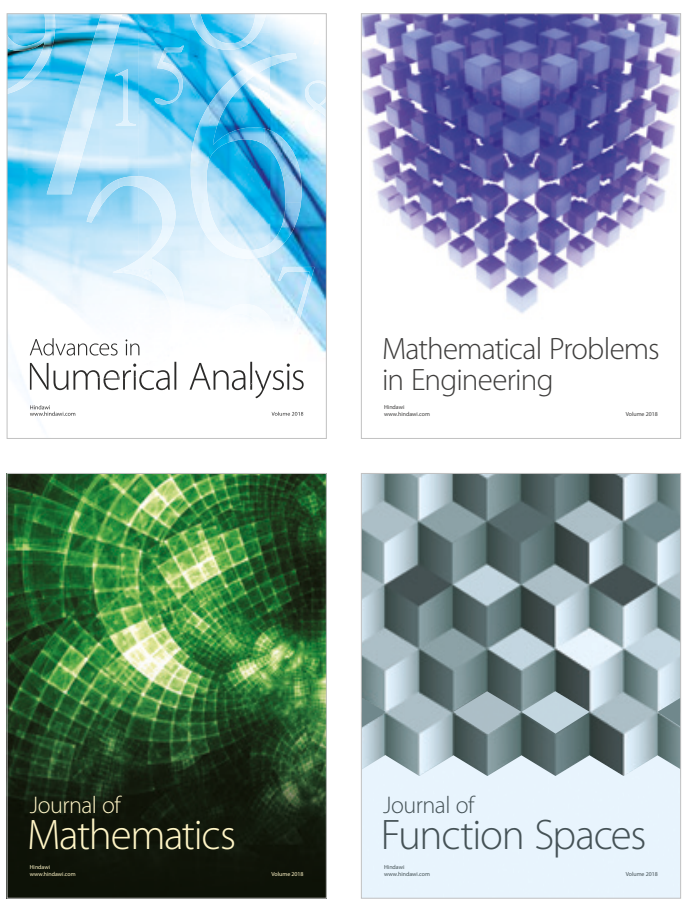

Mathematical Problems in Engineering

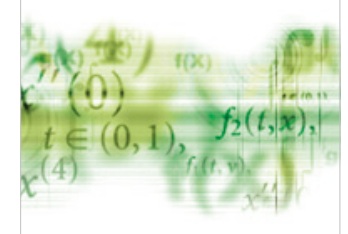

International Journal of

Differential Equations

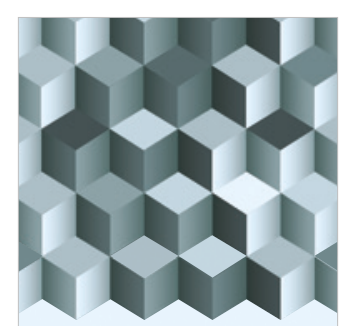

Journal of

Function Spaces

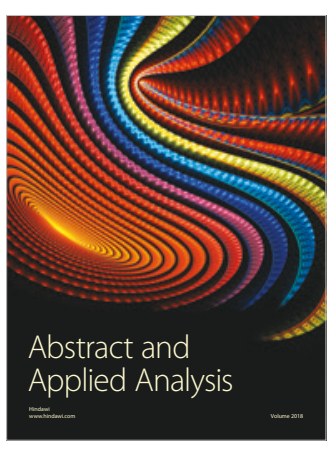

The Scientific

World Journal

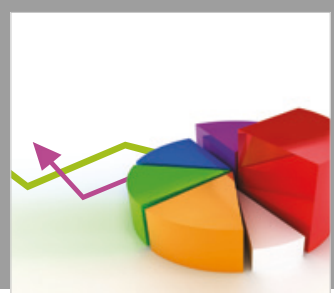

Journal of

Probability and Statistics
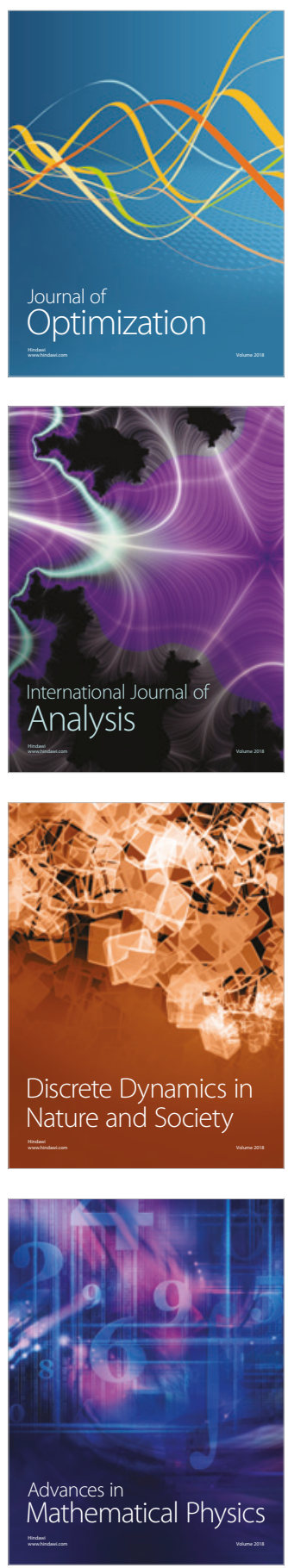